\title{
Detecting matter effects in long baseline experiments
}

\author{
Debajyoti Choudhury $^{1}$ and Anindya Datta ${ }^{2}$ \\ ${ }^{1}$ Department of Physics and Astrophysics, University of Delhi, Delhi 110 00\%, India. \\ ${ }^{2}$ INFN, Sezione di Roma; Dip. di Fisica, Universita La Sapienza, I-00185, Rome, Italy.
}

\begin{abstract}
Experiments strongly suggest that the flavour mixing responsible for the atmospheric neutrino anomaly is very close to being maximal. Thus, it is of great theoretical as well as experimental importance to measure any possible deviation from maximality. In this context, we reexamine the effects of matter interactions in long baseline neutrino oscillation experiments. Contrary to popular belief, the muon neutrino survival probability is shown to be quite sensitive to matter effects. Moreover, for moderately long baselines, the difference between the survival probilities for $\nu_{\mu}$ and $\bar{\nu}_{\mu}$ is shown to be large and sensitive to the deviation of $\left|U_{\mu 3}\right|$ from maximality. Performing a realistic analysis, we demonstrate that a muon-storage ring $\nu$-source alongwith an iron calorimeter detector can measure such deviations. (Contrary to recent claims, this is not so for the NuMIMINOS experiment.) We also discuss the possible correlation in measuring $U_{\mu 3}$ and $U_{e 3}$ in such experiment.
\end{abstract}

\section{Introduction}

Neutrino masses and mixings continue to intrigue us, despite the continued efforts of many recent experiments $[1-6]$. We do know that the muon-neutrino $\left(\nu_{\mu}\right)$ mixes almost maximally $\left(\sin ^{2} 2 \theta_{\mu 3}>0.92\right.$ at $90 \%$ C.L) with another species $\nu_{3}$ (which could be identified with the tauneutrino, $\left.\nu_{\tau}\right)$ and that the mass splitting $\left|\delta m_{32}^{2}\right| \sim 2 \times 10^{-3} \mathrm{eV}^{2}$. Furthermore, the electronneutrino $\left(\nu_{e}\right)$ mixes with a combination of $\nu_{\mu}$ and $\nu_{\tau}$ with a similarly large angle $\theta_{e 2} \simeq 30^{\circ}$ but a far smaller mass splitting $\left(\delta m_{21}^{2} \sim 7 \times 10^{-5} \mathrm{eV}^{2}\right)$. And, finally, the third mixing angle $\theta_{13}$ is constrained, by the CHOOZ experiment [3], to be small. What we do not know though are the sign of $\delta m_{32}^{2}$, the precise value of $\theta_{13}$ and the extent to which $\theta_{\mu 3}$ may, if at all, differ from maximality. Since such knowledge is obviously essential to the formulation of a theory of neutrino flavours, the importance of precise determinations cannot but be underemphasised. Neutrino oscillations provide just the proper platform for such an exercise.

As is well known, during passage through dense matter, various amplitudes for neutrino oscillations may be magnified to a great degree [7] thereby rendering them measurable with relative ease. While propagating in a medium, all the three neutrino flavours interact with matter via neutral curent (NC) interactions with equal strength. Those of the electron flavour, $\stackrel{(-)}{\nu_{e}}$, have, in addition, a extra charged current interaction as well. Since it is this extra

\footnotetext{
${ }^{1}$ On leave from Harish-Chandra Research Institute, Allahabad, India.

${ }^{2}$ E-mail: debchou@physics.du.ac.in, debchou@mail.cern.ch, Anindya.Datta@roma1.infn.it
} 
interaction that provides flavour-dependence, all matter-induced effects in neutrino oscillations (including those in the $\nu_{\mu}-\nu_{\tau}$ sector) are proportional to the size of the relevant mixing with $\nu_{e}$.

Recent theoretical advances in the quest of producing very high intensity muon sources engender optimism regarding a future neutrino factory wherein an intense beam of muons is to be accelerated to a not too high energy and stored in a storage ring with a straight section directed towards a neutrino detector. Muons would decay in this straight section thereby producing high intensity neutrino beams (both of electron- and muon-types) that are highly collimated in the direction of the decaying muons [8]. The efficacy of muon storage ring neutrino sources in performing precision measurements of neutrino parameters has already been discussed in the literature [9]. The advantages over neutrino experiments with conventional neutrino beams (arising from $\pi^{ \pm}$decay) are manifold: $(i)$ a precise knowledge of the $\nu_{\mu}$ and $\bar{\nu}_{e}$ fluxes helps reduce the systematic errors; (ii) assuming a $\mu$-beam of, say, $20 \mathrm{GeV}$ energy, such neutrinos have, on the average, energies higher than those of conventional neutrino beams thereby increasing the $\nu$ cross-section at the detector; and (iii) the aforementioned collimation.

Previous studies of the matter effect in Ref. [10] at a long base line experiment were concentrated mainly on the measurement of the rate of wrong sign muon events, which, in turn is proportional to the transition probablity $P_{\nu_{e} \rightarrow \nu_{\mu}}$. Such a measurement would enable a determination of (or constraining of) $\sin \theta_{13}$ provided independent and precise measurements of the mixing angle $\theta_{23}$ and mass-square difference $\delta m_{31}^{2}$ exist. Possible measurements of neutrino mixing parameters from long baseline experiments using conventional neutrino beams as well as from a neutrino factory, and a water Čerenkov detector has also been discussed in Ref. [11].

In the present article, we demonstrate that a sizable matter effect is also evinced by a measurement of the $\nu_{\mu}$ survival probability. In addition, such a measurement has the potential of detecting possible deviations from maximality of the mixing angle responsible for the atmospheric neutrino anomaly. In a recent study [12], the presence of large matter effect in $\nu_{\mu}-\nu_{\tau}$ oscillation as well as in $\nu_{\mu}$ survival probability has been stressed. Although there is a large matter effect present in $\nu_{\mu}-\nu_{\tau}$ sector, measurement of the same is difficult due to both the lower detection efficiency for the tau-events as well as the need for a more sophisticated detector.

Whereas much of our analysis would be source and detector independent, we also choose to examine the feasibility of making such meaurements in the context of a realistic experimental setup. To be concrete, we choose a $50 \mathrm{kT}$ Iron detector, with detection and charge discrimination capability for muons provided by a magnetic field. Such a detector was proposed for Gran Sasso (mONOLITH) [13] and, more recently, for a location in India (INO) [14]. The latter is contemplated primarily as a detector for atmospheric neutrinos and also as an end detector for a future neutrino factory beam. A muon detection threshold of $2 \mathrm{GeV}$ [14] has been used in our calculation. As for the baseline, we illustrate our results for two particular choices. The first one coresponds to a distance from JHF to INO $(\sim 5000 \mathrm{Km})$ and the other one corresponds to even a longer baseline, from FERMILAB to INO $(\sim 10000 \mathrm{Km})$. We will also compare results with a baseline of $732 \mathrm{Km}$, corresponding to the Fermilab-MINOs distance.

The plan for the rest of the article is as follows. In section 2, we discuss neutrino oscillation in the presence of matter. We also define the asymmetry of $\mu^{+}$and $\mu^{-}$events and explain its correlation to $U_{e 3}$ and $U_{\mu 3}$. We present the expressions for the asymmetry for two different aproximations: when matter effect is small compared to $\frac{\delta m_{32}^{2}}{2 E_{\nu}}$ i.e for short base line experiments like MINOs [15] and also when the matter term is comparable to the $\frac{\delta m_{32}^{2}}{2 E_{\nu}}$ i.e., for long base line 
experiments. In section 3, we briefly review and reexamine the proposal, in a recent article [15], to measure matter effect and the above correlations in the context of the MINOS experiment. Section 4 deals with the expectations at lomg baseline experiments, both for the $\nu_{\mu}$ survival probability as well as for the $\nu_{e}$ to $\nu_{\mu}$ transition probability. An analysis of the findings in the context of a realistic experimental situation is effected in Section 5. Finally, we conclude in Section 6.

\section{Neutrino Oscillation in presence of matter}

We begin by reviewing the passage of neutrino through matter. While analytical results are possible, the exact answers are cumbersome and not very useful. However, it is useful to consider approximations such as a constant density profile for the earth. While such an assumption is obviously not a very accurate one, especially when neutrinos traverse a longer distance through the earth, the approximation serves to give us some physical insights to the problem we are dealing with. Furthermore, results such as those we shall obtain are still applicable if the matter density within the earth may be approximated to be piecewise constant. It must be kept in mind though that the quantitative results presented in this article are obtained not by means of such an approximation, but by explicitly accounting for the varying density profile of the earth using the Preliminary Reference Earth Model (PREM) [16].

As with the quark sector, neutrino flavour states can be expressed in terms of mass eigenstates through a relation of the form

$$
\left|\nu_{\alpha}\right\rangle=\sum_{i} U_{\alpha i}\left|\nu_{i}\right\rangle
$$

where $U$ is a $3 \times 3$ unitary matrix known as the Pontecorvo-Maki-Nakagawa-Sakata mixing matrix. In the absence of Majorana phases (assumed to be so henceforth) ${ }^{3}, U$ can be parametrized in terms of three mixing angles and a $C P$-violating phase $\delta_{\mathrm{CP}}$, viz.

$$
U=\left(\begin{array}{ccc}
c_{12} c_{13} & s_{12} c_{13} & s_{13} e^{-i \delta_{\mathrm{CP}}} \\
-c_{23} s_{12}-s_{23} s_{13} c_{12} e^{i \delta_{\mathrm{CP}}} & c_{23} c_{12}-s_{23} s_{13} s_{12} e^{i \delta_{\mathrm{CP}}} & s_{23} c_{13} \\
s_{23} s_{12}-c_{23} s_{13} c_{12} e^{i \delta_{\mathrm{CP}}} & -s_{23} c_{12}-c_{23} s_{13} s_{12} e^{i \delta_{\mathrm{CP}}} & c_{23} c_{13}
\end{array}\right),
$$

with $c_{i j} \equiv \cos \theta_{i j}$ and $s_{i j} \equiv \sin \theta_{i j}$. In vacuum, neutrino oscillations are then governed by $U$ and the two independent differences in the squares of the neutrino masses, $\delta m_{i j}^{2} \equiv m_{i}^{2}-m_{j}^{2}$. In the rest of our analysis, we will make a further simplifying assumption of $\delta_{\mathrm{CP}}=0$. With the above parametrization of $U$, experimental results can be used to constrain the mixing angles $\theta_{i j}$ and the mass-square differences. Measurements of the solar neutrino fluxes, augmented by the KamLAND reactor data give

$$
0.30<\tan ^{2} \theta_{12}<0.64, \quad 5.4 \times 10^{-5} \mathrm{eV}^{2}<\delta_{21}<9.4 \times 10^{-5} \mathrm{eV}^{2}
$$

with the best-fit values given by $\tan ^{2} \theta_{12}=0.40$ and $\delta m_{21}^{2}=6.9 \times 10^{-5} \mathrm{eV}^{2}$. Experiments with atmospheric neutrinos (as well as the K2K experiment) suggest, on the other hand, that

$$
\sin ^{2} 2 \theta_{23}>0.86, \quad 1.4 \times 10^{-3} \mathrm{eV}^{2}<\left|\delta m_{32}^{2}\right|<5.1 \times 10^{-3} \mathrm{eV}^{2}
$$

\footnotetext{
${ }^{3}$ Any Majorana phase in the PMNS matrix cannot be probed by an oscillation experiment.
} 
with the best-fit value being $\left|\delta m_{32}^{2}\right|=2.0 \times 10^{-3} \mathrm{eV}^{2}$. And, finally, the negative result of the CHOOz experiment implies that

$$
\sin ^{2} \theta_{13}<5 \times 10^{-2} \quad \text { at } 99.73 \% \text { C.L. }
$$

Note that,

- unlike in the case for $\delta m_{21}^{2}$, the sign of $\delta m_{32}^{2}$ is undetermined;

- since $\left|\delta m_{32}^{2}\right| \approx\left|\delta m_{31}^{2}\right| \gg\left|\delta m_{21}^{2}\right|^{4}$, the last-mentioned (as well as the solar mixing angle $\theta_{12}$ ) plays only a subservient role in $\nu_{\mu}$ oscillations;

- while $\left|U_{\mu 3}\right|$ is constrained to be close to $1 / \sqrt{2}$, it is still allowed to be non-maximal.

Working in the limit of a vanishingly small $\delta m_{21}^{2}$, all the expressions for the oscillation/survival probabilities can be expressed in terms of just two of the mixing angles ${ }^{5}$, viz. $\theta_{23}$ and $\theta_{13}$. For a $\nu_{\mu}\left(\bar{\nu}_{\mu}\right)$ of energy $E_{\nu}$ travelling a distance $L$ in vacuum, the survival probability is then given by

$$
P_{\mu \mu}^{\mathrm{vac}}=P_{\bar{\mu} \bar{\mu}}^{\mathrm{vac}}=1-4\left|U_{\mu 3}\right|^{2}\left(1-\left|U_{\mu 3}\right|^{2}\right) \sin ^{2}\left(\Delta_{31} L / 2\right),
$$

where,

$$
U_{e 3}=s_{13}, \quad U_{\mu 3}=c_{13} s_{23}, \quad \Delta_{31} \equiv \frac{\delta m_{31}^{2}}{2 E_{\nu}}
$$

In the presence of matter, the electron (anti-)neutrino sees an additional potential corresponding to charged current interactions, and the effective Hamiltonian is given by

$$
H=U\left(\begin{array}{ccc}
0 & 0 & 0 \\
0 & \Delta_{21} & 0 \\
0 & 0 & \Delta_{31}
\end{array}\right) U^{\dagger}+\left(\begin{array}{ccc}
A & 0 & 0 \\
0 & 0 & 0 \\
0 & 0 & 0
\end{array}\right)
$$

where $A \equiv \pm \sqrt{2} G_{F} N_{e}$ for $\stackrel{(-)}{\nu}$, with $N_{e}$ being the instantaneous electron density of the matter.

The survival probablities suffer a consequent modification. While the full expressions are cumbersome, it is instructive to look at a simplified form obtainable for a matter density small enough that $A$ can be treated as a perturbation in the effective Hamiltonian. In this limit, following Ref. [15],

$$
\begin{aligned}
P_{\mu \mu(\bar{\mu} \bar{\mu})} & \simeq P^{\mathrm{vac}} \pm\left(\Delta P_{\mu \mu} / 2\right)+\mathcal{O}\left(\widetilde{A}^{2}\right) \\
\Delta P_{\mu \mu} & \simeq 4 \widetilde{A}\left|U_{e 3}\right|^{2}\left|U_{\mu 3}\right|^{2}\left(1-2\left|U_{\mu 3}\right|^{2}\right)\left[4 \sin ^{2} \Psi-\Psi \sin (2 \Psi)\right]+\mathcal{O}\left(\widetilde{A}^{2}\right)
\end{aligned}
$$

where

$$
\widetilde{A} \equiv \frac{A}{\Delta_{31}} \quad \text { and } \quad \Psi \equiv \frac{\Delta_{31} L}{2} .
$$

\footnotetext{
${ }^{4}$ From now on we will denote this large mass-square difference by $\delta m_{31}^{2}$

${ }^{5}$ While the analytical expressions below have been derived under this approximation, all of the numerical analysis has been performed using the full expression for $U$ and a non-zero value for $\delta_{12}$.
} 
The asymmetry $\Delta P_{\mu \mu}$ is a measure of the matter effect and that it should be proportional to both $\widetilde{A}$ and $\left|U_{\mu 3}\right|^{2}$ is obvious. Note also that the $\nu_{\mu}$ has the same interaction with matter as the $\nu_{\tau}$ with which it primarily mixes and any matter effect can only seep in through a mixing with the $\nu_{e}$. This, then, accounts for the overall factor of $\left|U_{e 3}\right|^{2}$. All of these three proportionalities quite independent of the approximation, and, in fact, are exact results. The factor $\left(1-2\left|U_{\mu 3}\right|^{2}\right)$, however, is but a consequence of the approximation of a small matter term $\widetilde{A}$ and is applicable only for neutrinos traversing small base lines. Since this factor vanishes identically for a maximal mixing in the $\nu_{\mu}-\nu_{\tau}$ sector, this matter-induced asymmetry is potentially a sensitive probe of the maximality of $U_{\mu 3}$. This has already been pointed out [15] and examined in the context of the MINOS experiment. In the next section we will briefly reexamine their claims.

Convoluting eqn.(9) with the $E_{\nu}$-dependent flux and the detection efficiency of a given detector, one would obtain the number of events and thereby the event asymmetry

$$
\Delta N=N_{\nu_{\mu}}-N_{\bar{\nu}_{\mu}} \equiv N_{-}-N_{+} .
$$

The latter could then be used to place contraints in the $\left|U_{\mu 3}\right|-\left|U_{e 3}\right|$ plane. Of course, in a realistic case, the effects of a varying matter density has to be taken into account as well and this we do include in our analysis. With the effect being proportional to $\widetilde{A}$, it will be magnified when the (anti-)neutrino beam traverses regions of high density, or in other words, the core of the earth. Note, however, that a large $\widetilde{A}$ results in a breakdown of the simplified form given in eq.(9) and that the full expression (or, at least, a different approximation) needs to be used.

The exact expression (for an arbitrarily large but constant density) for the survival probability is given by

$$
\begin{aligned}
P_{\mu \mu}=1-4 s_{23}^{2} & \left(c_{23}^{2} s_{\theta_{m}}^{2} \sin ^{2}\left[\frac{\Delta_{31} L}{4}(1+\widetilde{A}-\mathcal{D})\right]\right. \\
& \left.+s_{23}^{2} s_{\theta_{m}}^{2} c_{\theta_{m}}^{2} \sin ^{2} \frac{\Delta_{31} L \mathcal{D}}{2}+c_{23}^{2} c_{\theta_{m}}^{2} \sin ^{2}\left[\frac{\Delta_{31} L}{4}(1+\widetilde{A}+\mathcal{D})\right]\right),
\end{aligned}
$$

where

$$
\mathcal{D} \equiv \sqrt{1+\widetilde{A}^{2}-2 \widetilde{A} \cos 2 \theta_{13}}, \quad \text { and } \quad \theta_{m} \equiv \frac{1}{2} \tan ^{-1} \frac{\sin 2 \theta_{13}}{\cos 2 \theta_{13}-\widetilde{A}}
$$

We now perform a double expansion ${ }^{6}$ in $\left|U_{e 3}\right|$ as well as $\beta \equiv \frac{1}{2}-\left|U_{\mu 3}\right|^{2}$ while allowing for any value for $\widetilde{A}$. Since both the expansion parameters are small, we may retain terms only upto, say, the fourth order without any loss of accuracy. Then, for the survival probability, we

\footnotetext{
${ }^{6}$ This is similar in spirit to, though not the same as, the expansion performed in Ref. [17].
} 
have

$$
\begin{aligned}
P_{\mu \mu} & =\left(1-4 \beta^{2}\right) c_{\Psi}^{2} \\
+ & \frac{\left|U_{e 3}\right|^{2}}{(1-\widetilde{A})^{2}}\left[\left\{s_{\Psi}^{2}-s_{\Omega}^{2}-s_{\Theta}^{2}-\widetilde{A}(1-\widetilde{A}) \Psi s_{2 \Psi}\right\}-4 \beta\left\{(1-\widetilde{A})^{2} s_{\Psi}^{2}-s_{\Omega}^{2}\right\}\right. \\
& \left.+4 \beta^{2}\left\{\left(1-4 \widetilde{A}+2 \widetilde{A}^{2}\right) s_{\Psi}^{2}+s_{\Omega}^{2}-s_{\Theta}^{2}+\frac{\widetilde{A}}{2}(1-\widetilde{A}) \Psi s_{2 \Psi}\right\}\right] \\
+ & \frac{\left|U_{e 3}\right|^{4}}{(1-\widetilde{A})^{4}}\left[\left(1-6 \widetilde{A}+\widetilde{A}^{4}-4 \widetilde{A}^{3}+5 \widetilde{A}^{2}\right) s_{\Psi}^{2}+\left(2 \widetilde{A}+\widetilde{A}^{2}\right) s_{\Omega}^{2}\right. \\
& \quad-\Omega^{2}(1-\widetilde{A})^{2} c_{2 \Psi}+\left(-1+6 \widetilde{A}-\widetilde{A}^{2}\right) s_{\Theta}^{2} \\
+ & \left.\quad+\left(1-\widetilde{A}^{2}\right) \Omega s_{2 \Psi}+(1-\widetilde{A}) \Omega\left(s_{2 \Omega}+2 s_{2 \Theta}\right)\right] \\
+ & \mathcal{O}\left(\left|U_{e 3}\right|^{4} \beta,\left|U_{e 3}\right|^{3} \beta^{2},\left|U_{e 3}\right|^{2} \beta^{3},\left|U_{e 3}\right| \beta^{4}\right),
\end{aligned}
$$

where $s_{\alpha} \equiv \sin (\alpha), c_{\alpha} \equiv \cos (\alpha)$ with

$$
\Omega \equiv \frac{\Delta_{31} L \widetilde{A}}{2}=\frac{A L}{2}=\widetilde{A} \Psi \text { and } \quad \Theta \equiv \Omega-\Psi .
$$

With the survival probability for the antineutrinos being obtained by the replacement $\widetilde{A} \rightarrow-\widetilde{A}$ in eq.(14), we have, for the probability asymmetry

$$
\begin{aligned}
\frac{-\left(1-\widetilde{A}^{2}\right)^{3} \Delta P}{4 \widetilde{A}\left|U_{e 3}\right|^{2}\left|U_{\mu 3}\right|^{2}}= & \left(1-\widetilde{A}^{2}\right)\left[4 c_{\Psi}^{2} s_{\Omega}^{2}+\left(1-\widetilde{A}^{2}\right) \Psi s_{2 \Psi}-\frac{1+\widetilde{A}^{2}}{2 \widetilde{A}} s_{2 \Psi} s_{2 \Omega}\right] \\
+ & 2 \beta\left(1-\widetilde{A}^{2}\right)\left[-4 s_{\Psi}^{2} s_{\Omega}^{2}+\left(1-\widetilde{A}^{2}\right) \Psi s_{2 \Psi}+\frac{1+\widetilde{A}^{2}}{2 \widetilde{A}} s_{2 \Psi} s_{2 \Omega}\right] \\
+ & \left|U_{e 3}\right|^{2}\left[\frac{4}{1-\widetilde{A}^{2}}\left\{\left(1+11 \widetilde{A}^{2}+2 \widetilde{A}^{4}\right) s_{\Psi}^{2} c_{\Omega}^{2}+\widetilde{A}^{2}\left(3-\widetilde{A}^{2}\right) c_{\Psi}^{2} s_{\Omega}^{2}\right\}\right. \\
& +\Psi\left\{2 \widetilde{A}^{2}\left(1-\widetilde{A}^{2}\right)^{2} \Psi c_{2 \Psi}-\left(1+6 \widetilde{A}^{2}+\widetilde{A}^{4}\right) s_{\Psi} c_{\Psi}\right\} \\
& +s_{2 \Psi}\left\{2\left(1+3 \widetilde{A}^{2}\right) \Psi c_{2 \Omega}-\frac{1-16 \widetilde{A}^{2}-\widetilde{A}^{4}}{4 \widetilde{A}} s_{2 \Omega}\right\} \\
& \left.-\left(3+\widetilde{A}^{2}\right) \Omega s_{2 \Omega}\left(3-4 s_{\Psi}^{2}\right)\right] \\
+ & \mathcal{O}\left(\left|U_{e 3}\right|^{2} \beta,\left|U_{e 3}\right| \beta^{2}, \beta^{3}\right) .
\end{aligned}
$$

Note that

- The asymmetry $\Delta P$ continues to be proportional to each of $\widetilde{A},\left|U_{e 3}\right|^{2}$ and $\left|U_{\mu 3}\right|^{2}$, but not to $\left(1-2\left|U_{\mu 3}\right|^{2}\right)$.

- $\lim _{\widetilde{A} \rightarrow 0} s_{\Omega} / \widetilde{A}=\Psi$ and hence the right hand side of eq.(16) is finite in this limit.

- To $\mathcal{O}(\widetilde{A})$, the expressions for both $P_{\mu \mu}$ and $\Delta P$ coincide with those of eq. $(9)$. 
- The strength of the $\mathcal{O}(1)$ term on the r.h.s. of eq.(16) — which vanishes as $\widetilde{A}^{2}$ for small $\widetilde{A}$ - gives a measure of the violation of the $\left(1-2\left|U_{\mu 3}\right|^{2}\right)$ proportionality of $\Delta P$.

- While we have performed the expansion upto $\mathcal{O}\left(\left|U_{e 3}\right|^{4}\right)$, for all practical purposes it suffices to consider only upto $\mathcal{O}\left(\left|U_{e 3}\right|^{2}\right)$.

- The apparent singularities in eqs.(14\& 16) are not real ones with

$$
\begin{aligned}
\lim _{\widetilde{A} \rightarrow 1} P_{\mu \mu} & =c_{\Psi}^{2}+4 \beta^{2} s_{\Psi}^{2} \\
& +\left|U_{e 3}\right|^{2}\left[\Psi\left(s_{2 \Psi}-2 \Psi c_{\Psi}^{2}\right)+4 \beta\left(\Psi^{2}-s_{\Psi}^{2}\right)+4 \beta^{2}\left\{2\left(1-\Psi^{2}\right) s_{\Psi}^{2}-\Psi s_{2 \Psi}\right\}\right] \\
& +\left|U_{e 3}\right|^{4} \Psi^{2}\left[s_{\Psi}^{2}+\frac{\Psi^{2}}{3}\left(3+2 s_{\Psi}^{2}\right)-\frac{4}{3} \Psi s_{2 \Psi}\right]
\end{aligned}
$$

and

$$
\begin{aligned}
\lim _{\widetilde{A} \rightarrow 1} \frac{-\Delta P}{\left|U_{e 3}\right|^{2}\left|U_{\mu 3}\right|^{2}}= & 2\left[2 \Psi^{2} c_{\Psi}^{2}-\Psi s_{\Psi} c_{\Psi}-\frac{s_{2 \Psi}^{2}}{4}\right]+\beta\left[-\Psi s_{2 \Psi}+\left(5-8 \Psi^{2}\right) s_{\Psi}^{2}+s_{2 \Psi}^{2}\right] \\
+ & \left|U_{e 3}\right|^{2}\left[-s_{2 \Psi}^{2}-\Psi^{2}\left(\frac{3}{2}+s_{\Psi}^{2}\right)-\frac{2 \Psi^{4}}{3}\left(3+2 c_{\Omega}^{2}\right)\right. \\
& \left.+\frac{4 \Psi^{3}}{3} s_{2 \Psi}+\Psi s_{2 \Psi}\left(\frac{5}{8}-s_{\Psi}^{2}\right)\right] .
\end{aligned}
$$

\section{Can the NuMI-MINOS Combine See This?}

With the Fermilab Main Injector serving as a neutrino source, the MINOS detector [18] at the Soudan Mine offers a baseline of $732 \mathrm{~km}$ and hence a possible setup for making such a measurement. This was investigated by Choubey and Roy in Ref. [15]. Working with a $\nu_{\mu}$ exposure of $16 \times 10^{20}$ primary protons on target, they conclude that a significant rate asymmetry $(\Delta N)$ can be observed for moderate values of $\left(1-2\left|U_{\mu 3}\right|^{2}\right)$ and $\left|U_{e 3}\right|^{2}$.

However, while their idea was an interesting one and the formalism essentially correct, a reestimation of the rate asymmetry using the same set of inputs reveals some discrepancies. While we agree with Ref. [15] on the magnitude of $\Delta N$ for all the different cases of inputs parameters, we completely disagree on the issue of the error bars. Even on using an optimistic indicator of the background fluctuation, namely $\sqrt{N_{\mu}+N_{\bar{\mu}}}$, we find that, for the abovementioned luminosity, the statistical error bars are large enough for each of the rate asymetries in Ref. [15] to be consistent with zero.

Thus, to obtain a result of any statistical significance would need the flux (or, equivalently, the detector size) to be increased manifold (see Fig. 1). Such a large scaling, unfortunately, is not feasible with the present experimental facility. Note, in addition, that this projection altogether neglects the systematic uncertainties, and consequently is already too optimistic. In reality, several uncertainties are involved in the translation between the visible energy observed at a detector and the energy of the neutrino to which the event is ascribed. These include the uncertainties in the assumed final state multiplicities, the scattering or absorption of the secondary particles etc. In a recent paper [19], the MINER $\nu$ A collaboration, estimates the size of the corresponding systematic effects at MINOS-like experiments could be comparable to, or even dominate, the statistical errors. 


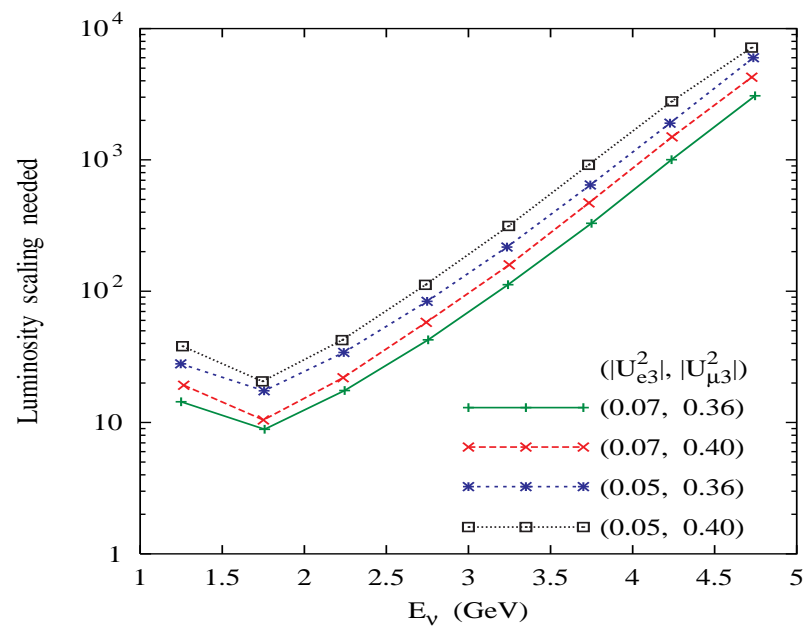

Figure 1: The minimal ratio by which the MINOS $\nu_{\mu}$ exposure $\left(16 \times 10^{20}\right.$ primary protons on target) needs to be scaled up to obtain $1 \sigma$ effects for each individual energy bin. The curves correspond to the four parameter points of Ref. [15]. Note that only the statistical fluctuations have been included in this analysis.

\section{The case for a very long baseline}

Having established that, contrary to the claims of Ref. [15], MINOS, in its current incarnation, is not sensitive to possible deviations of $\left|U_{\mu 3}\right|$ from maximality even for optimistic choices of parameters, it becomes interesting to investigate if any of the other proposed long-baseline experiments would do the job. And, in a similar vein, if even the luminosity scaling as described in the previous section would prove adequate. We start by investigating the second problem.

As has already been emphasised above, it is not only $\Delta P$ that is important, but also the average survival probability $P_{\mathrm{av}} \equiv\left(P_{\mu \mu}+P_{\bar{\mu} \bar{\mu}}\right) / 2$ for the latter controls the total number of events and hence the size of the statistical error (significance $\propto \Delta P_{\mu \mu} / \sqrt{2 P_{\mathrm{av}}}$ ). In Fig.2, we present both these quantities as function of neutrino energy for a baseline of $732 \mathrm{~km}$. The two choices of $\left(\left.U_{\mu 3}\right|^{2},\left|U_{e 3}\right|^{2}\right)$ correspond to the most (and least) optimistic sets of parameters used in Ref. [15].
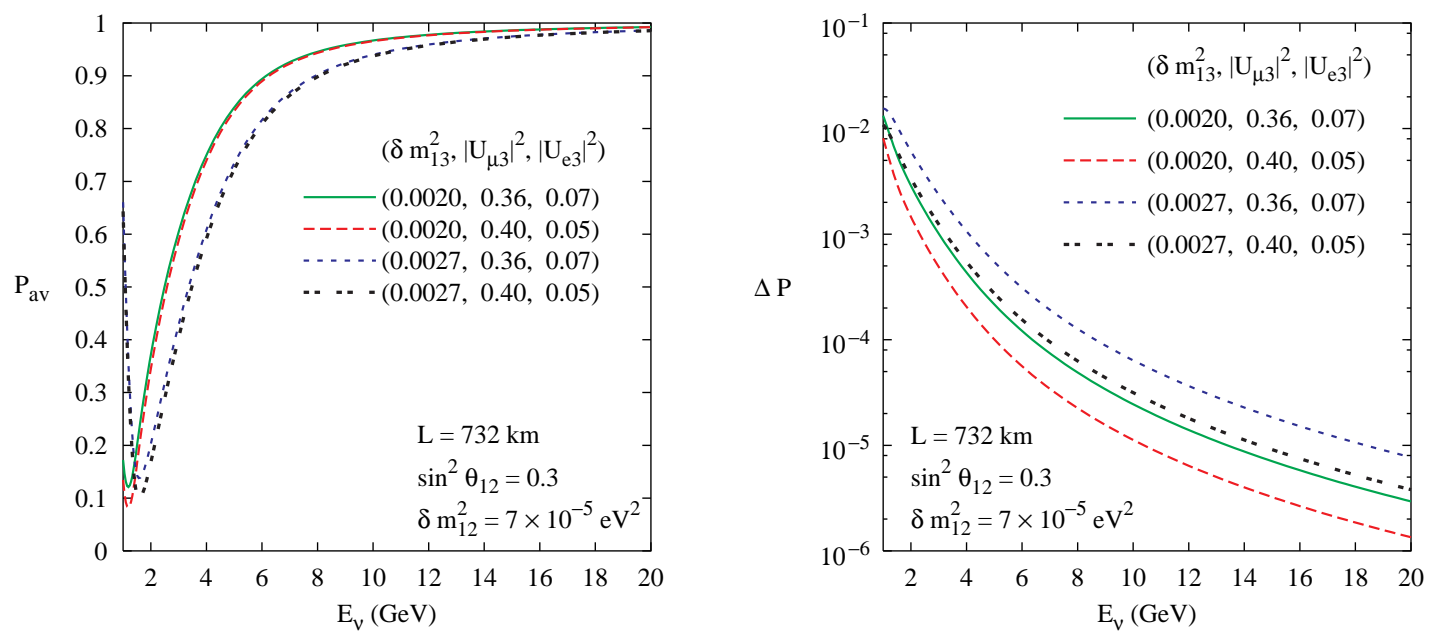

Figure 2: (a) The neutrino survival probability as a function of neutrino energy for a baseline $L=732 \mathrm{~km}$. The curves correspond to different combinations of $\delta m_{13}^{2}\left(\right.$ in $\left.\mathrm{eV}^{2}\right),\left|U_{\mu 3}\right|^{2}$ and $\left|U_{e 3}\right|^{2}$. (b) The corresponding difference in the $\nu_{\mu}$ and $\bar{\nu}_{\mu}$ survival probablities. 
It is abundantly clear that, for such a baseline, and for realistic choice of parameters, $\Delta P$ is very small, especially at larger neutrino energies. This was to be expected as a baseline of 732 $\mathrm{km}$ implies that the neutrino beam neither travels through a very dense matter core nor does it travel through a "dilute" segment long enough for the matter effect to build up sufficiently. Clearly, it is dangerous to claim a signal based on $\Delta P \lesssim 10^{-4}$, for such an act presupposes a very accurate knowledge of both the (anti-)neutrino cross sections as well as the density profile within the earth. Thus, one would have to concentrate only on neutrinos with relatively small energies $\left(E_{\nu} \lesssim 2 \mathrm{GeV}\right)$. Apart from the fact that the asymmetry is not too large even in this range $^{7}$, such a measurement also necessitates both a very good energy resolution as well as a low energy threshold. Furthermore, for such small energies, neutrino cross-section scales as $E_{\nu}^{2}$ and thus restricting ourselves to a small window is tantamount to rejecting a very large fraction of the events.
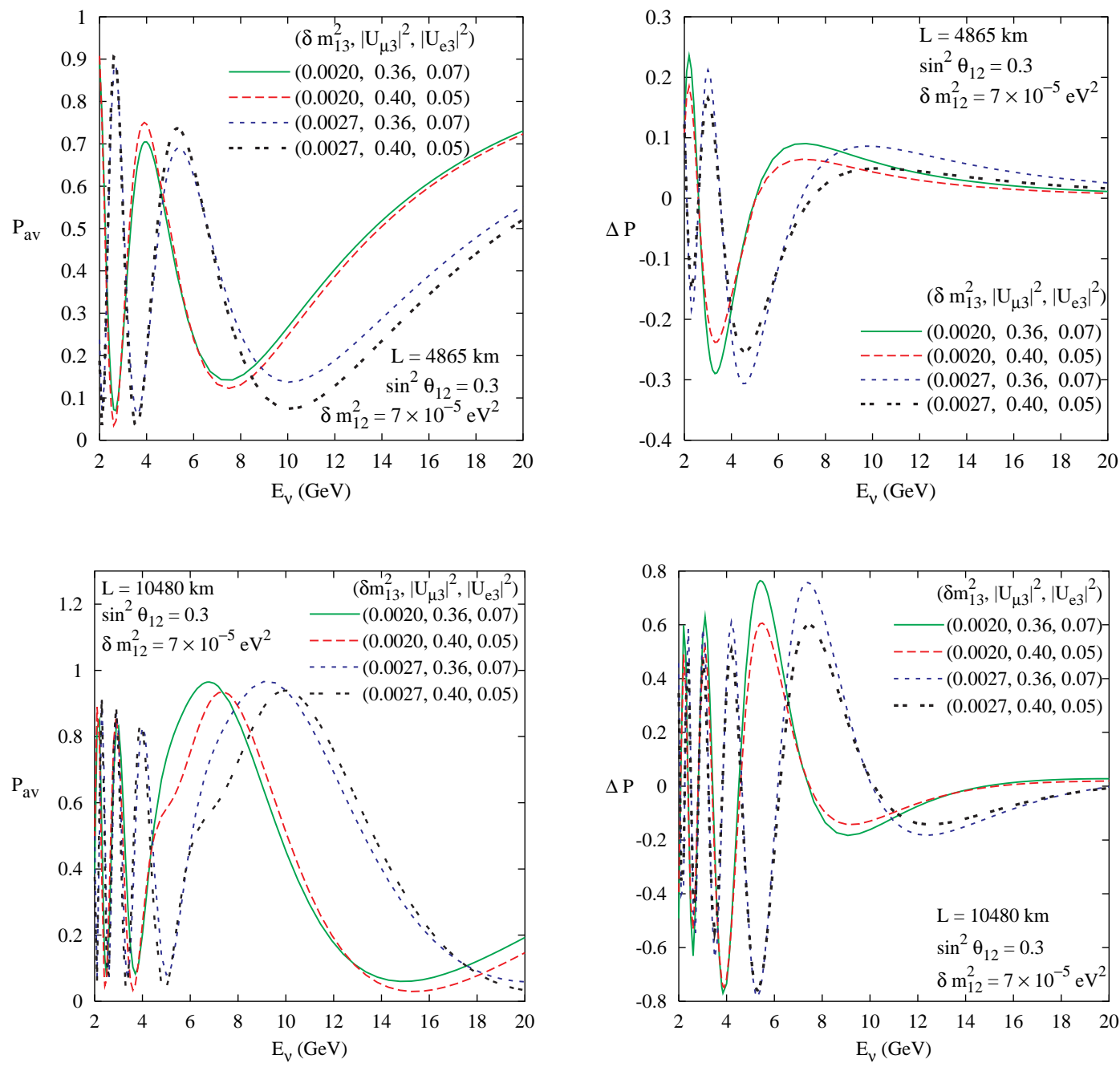

Figure 3: As in Fig.2, but for baselines $L=4865 \mathrm{~km}$ (upper panels) and $L=10480 \mathrm{~km}$ (lower panels) instead.

The situation, vis. a vis. the size of $\Delta P$, seems to improve dramatically with an increase in the baseline. For definiteness, we consider two particular values, namely $L=4865 \mathrm{~km}$ (the distance between the Japan Hadron Facility and the proposed Indian Neutrino Observatory

\footnotetext{
${ }^{7}$ In fact, it stands to be swamped by the systematic errors unless further experiments are performed [19].
} 
site at Rammam [14]) and $L=10480 \mathrm{~km}$ (the distance between Fermilab and INO). Since the matter term can no longer be treated as a perturbation, we desist from using the simplified form of eq.(9) and use the full expression for neutrino propagation in matter with the density profile being given by the Preliminary Reference Earth Model [16]. As Fig.3 amply demonstrates, for each of these values, $\Delta P$ is large over a wide range of $E_{\nu}$. In fact, for the larger of the two baselines $|\Delta P|$ can be as large as 0.8 for certain $E_{\nu}$ bins. Furthermore, unlike in the case of the $732 \mathrm{~km}$ baseline where $\Delta P$ was nearly monotonic in $E_{\nu}$, significant structure, including sign reversal, is shown. As can be easily appreciated, the latter feature has the potential of serving as a key experimental signature.

A further point of interest relates to the position of the maxima of the survival probabilities as well as the asymmetry. Clearly, a high resolution measurement of the same could, in principle, be used to determine $\delta m_{13}^{2}$.

Before we interpret Fig. 3 to imply that the asymmetry measurement in very long baseline experiments would be a sensitive probe of the deviation of $\left|U_{\mu 3}\right|$ from maximality, it should be realised that the latter is not the only source for a non-zero asymmetry. In fact, as has already been hinted at above, the higher order terms in $\widetilde{A}$ that were dropped while deriving eq.(9) are not proportional to $\left(1-2\left|U_{\mu 3}\right|^{2}\right)$. This can also be seen explicitly from eqs. $(14 \&$ 16) obtained assuming a constant density profile for earth. The numerical importance of such contributions naturally increases with the baseline, and has been explicitly displayed in Fig. 4. In fact, for the larger baseline, it is quite apparent that the bulk of the effect is due to such "higher-order" terms. Thus, it may be concluded that while a baseline of $\approx 5000 \mathrm{~km}$ may allow for a determination of $\left(1-2\left|U_{\mu 3}\right|^{2}\right)$ through measurements of the rate asymmetry, for baselines much longer than this the sensitivity reduces quite sharply, and a measurement of the maximality of $\left|U_{\mu 3}\right|$ is not very straightforward for ultra-long baselines.
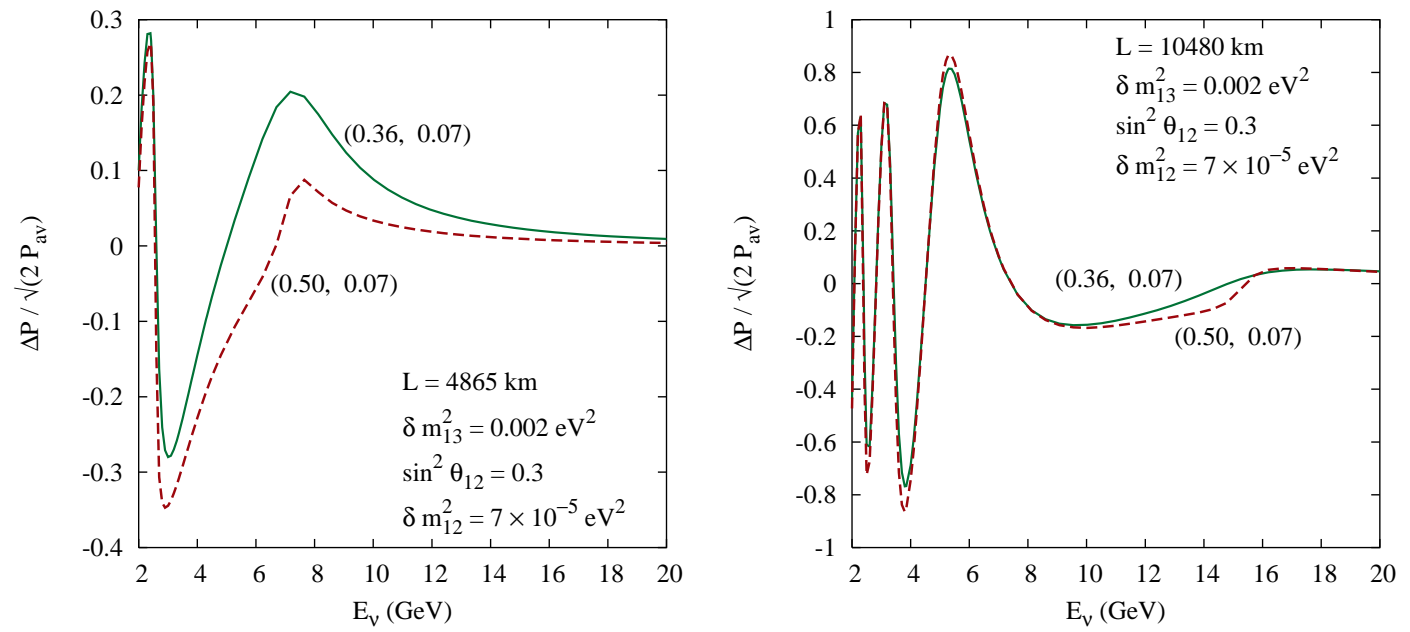

Figure 4: The normalized probability asymmetry for a baseline of (a) $4865 \mathrm{~km}$ and (b) 10480 $\mathrm{km}$. In each case, the solid (dashed) line refers to $\left|U_{\mu 3}\right|^{2}=0.36$ (0.50). Note that, according to the leading expression of eq.(9), the dashed curves should have coincided with the abscissa.

On the other hand, the asymmetry must remain proportional to $\left|U_{e 3}\right|^{2}$ even on the inclusion of $\widetilde{A}^{n}$ terms, for it is only through the interaction with the $\nu_{e}$ that matter effect truly enters $\nu_{\mu}-\nu_{\tau}$ oscillations. Thus, a baseline of $10480 \mathrm{~km}$ may still serve the purpose of affording a good measurement of $\left|U_{e 3}\right|$. In fact, since $U_{\mu 3}$ and $U_{e 3}$ are inextricably linked even for the case of the $4865 \mathrm{~km}$ baseline, such an independent measurement of the latter element has its own importance. 


\section{1 $\nu_{e} \rightarrow \nu_{\mu}$ oscillations}

With the matter effect playing such an important role in the survival probability for the muon (anti-)neutrino, it becomes interesting to consider its effect on $P\left(\nu_{e} \rightarrow \nu_{\mu}\right)$. For, a source that produces $\nu_{\mu}\left(\bar{\nu}_{\mu}\right)$ copiously, whether it be a beam dump or a muon storage ring, would also produce electron (anti-)neutrinos.
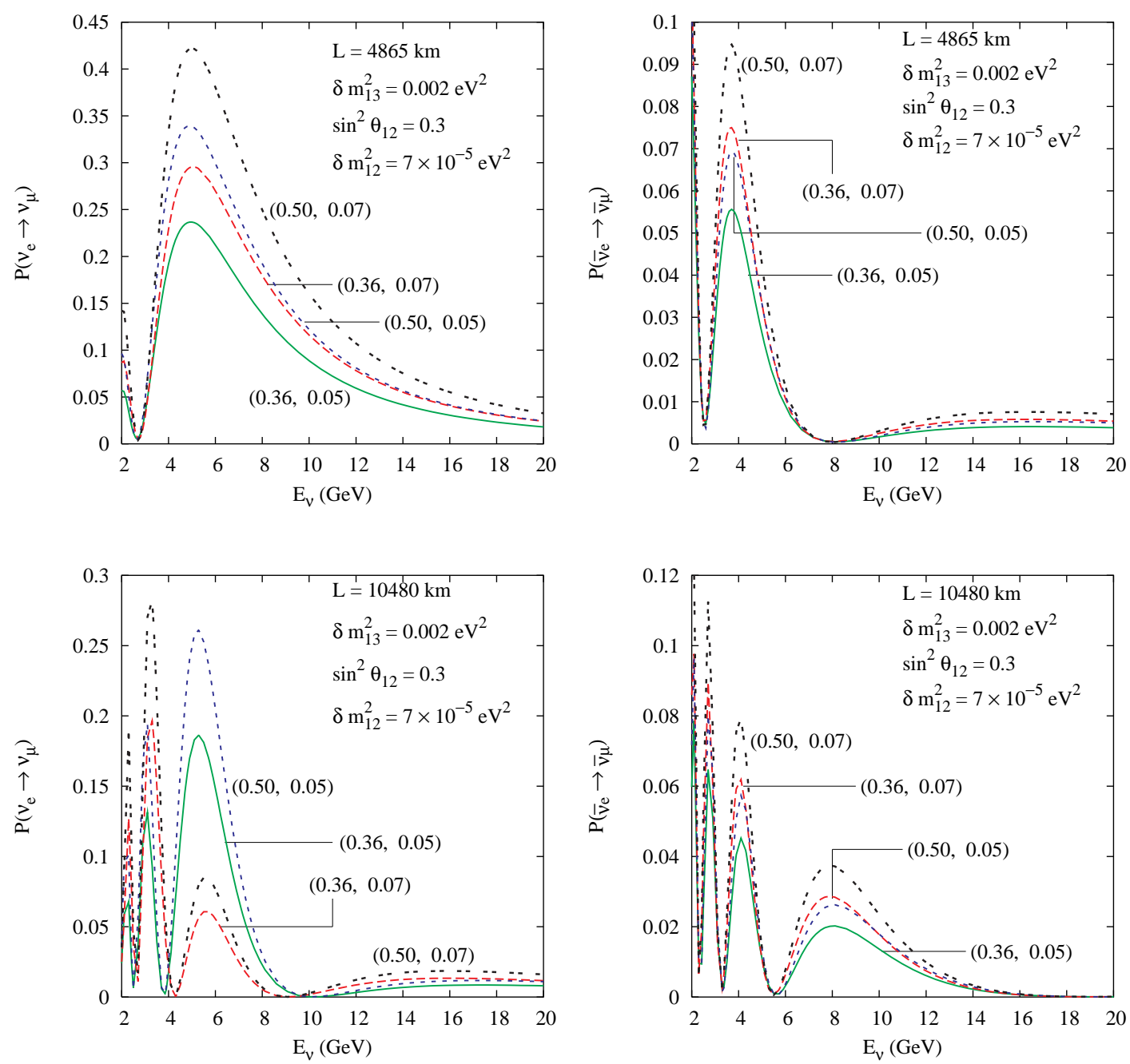

Figure 5: The energy dependence of the transition probability for $\nu_{e} \rightarrow \nu_{\mu}$ (left) and $\bar{\nu}_{e} \rightarrow \bar{\nu}_{\mu}$ (right) on passage through matter. The upper and lower panels correspond to baselines of 4865 and $10480 \mathrm{~km}$ respectively.

In Fig. 5, we present the probabilities for such transitions as a function of (anti-)neutrino energy. Several observations are in order:

- The transition probability $P\left(\nu_{e} \rightarrow \nu_{\mu}\right)$ can be quite sizable for parameter values that are still allowed by experimental data.

- For $E \gtrsim 3 \mathrm{GeV}$, we see that $P\left(\bar{\nu}_{e} \rightarrow \bar{\nu}_{\mu}\right) \lesssim P\left(\nu_{e} \rightarrow \nu_{\mu}\right)$ (except, of course, near the node at $\sim 8 \mathrm{GeV}$ for the $10480 \mathrm{~km}$ case). Coupled with the smaller detection efficiency for anti-neutrinos, this indicates that it would be more profitable to work with $\nu_{e}$ than $\bar{\nu}_{e}$.

- The transition probabilities have non-trivial dependence on both $U_{e 3}$ and $U_{\mu 3}$. Unlike 
in the case of the aforementioned asymmetry, these are not in general proportional to $\left|U_{e 3}\right|^{2}$.

- The measurement of this effect would thus lead to a constraint in the $U_{e 3}-U_{\mu 3}$ plane independent of that drawn from the asymmetry measurement.

\section{Event rate calculation from a storage ring neutrino source}

As we have already mentioned in the introductory section, the neutrino flux from a muon storage ring can be calculated very precisely. Starting with a $\mu^{-}$beam, the number of $\mu^{-}$ events in a far detector can be obtained by folding this flux with the survival probability $P_{\mu \mu}(L, E)$ and the charged current cross-section:

$$
N_{\mu}=N_{n} \int \sigma\left(\nu_{\mu}+N \rightarrow \mu^{-}+X\right) \frac{d N_{\nu}}{d E_{\nu_{\mu}}} P_{\mu \mu}(L, E) d E_{\nu_{\mu}}
$$

where $N_{n}$ is the total number of nucleons present in the fixed target. An analogous expression obtains for $\mu^{+}$events as well. Note though that the $\bar{\nu}_{e}$ from muon decay could also oscillate into $\bar{\nu}_{\mu}$ while traversing through the earth and result in muonic events. However, they result in wrong sign muons and thus can be easily distinguished in a magnetized detector. We shall revert back to them at a later stage.

To be specific, we shall consider a storage ring with $20 \mathrm{GeV}$ muons and a $50 \mathrm{kT}$ Iron calorimeter detector one such as the proposed MONOLITH [13] or the ICAL/INO experiment [14]. The projected energy threshold for muon detection is about $2 \mathrm{GeV}$ and the resolution is expected to be better than $0.5 \mathrm{GeV}$ over the entire range.

Before delving into the actual event profile, several points are in order.

- As has already been demonstrated in the previous section, the difference in the survival probalities $\left(\Delta P_{\mu \mu}\right)$ typically grows with the baseline. Thus the experimental sensitivity could be expected to increase with the baseline. (This is modulo the fact that the sensitivity of the asymmetry to $\left(1-2\left|U_{\mu 3}\right|^{2}\right)$ reduces for very large baselines, while the sensitivity to $\left|U_{e 3}\right|^{2}$ is retained.)

- On the other hand, for a fixed detector size, the effective solid angle subtended by the detector to the source decreases with increasing baseline. Consequently the neutrino flux goes down quadratically with the baseline, thereby resulting in a smaller number of events and hence larger statistical errors. This, for example, can be read off from Fig. 6, wherein we have displayed the number of events expected if the survival probability were to be unity.

- One obvious way to circumvent the latter problem is to start with higher luminosity. For our numerical results we then adopt the 'higher luminosity' option for the muon beam, namely $10^{20}$ muon decays per year.

- An alternative way to the same end would have been to start with a higher energy for the muon beam. For example, the neutrino beam from a $50 \mathrm{GeV}$ storage ring is collimated sufficiently enough to get a similar significance with even the 'low luminosity' option for 

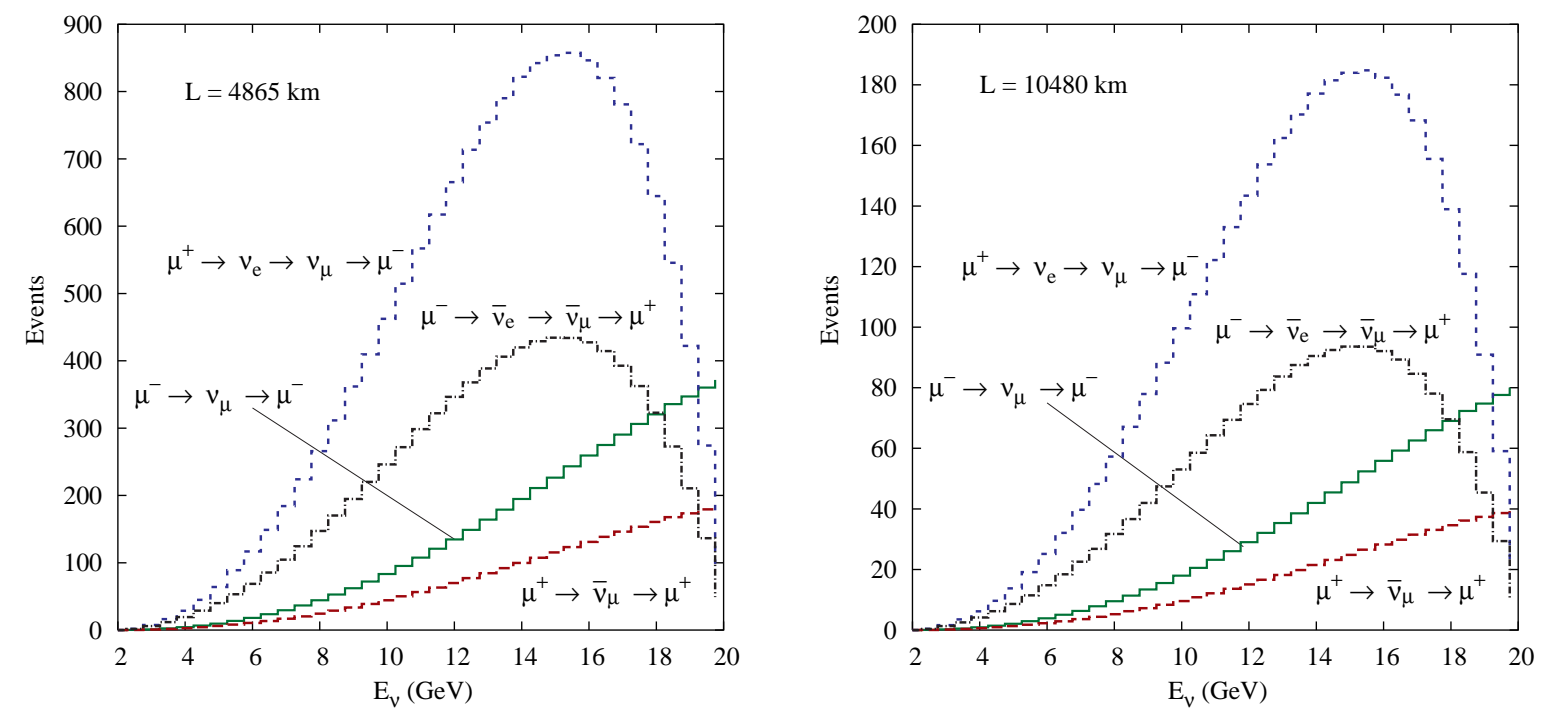

Figure 6: The number of events expected as a function of the (anti-)neutrino energy if the survival probability were to be unity. The source is a $20 \mathrm{GeV}$ muon storage ring with $10^{20}$ (anti-)muons decaying while the detector is a $50 \mathrm{kT}$ iron calorimeter with a energy threshold of 2 GeV for the $\mu^{ \pm}$[14]. The left (right) panel corresponds to a baseline of 4865 (10480) $\mathrm{km}$. Also shown are the events generated by the electron (anti-)neutrino assuming a transition probability of unity.

the storage ring (10 ${ }^{19}$ muon decays per year). However, we shall desist from using this option as such a machine is likely only as a second generation facility.

- As Fig. 3 amply demonstrates, for longer baselines such as the one we are considering, the survival probabilities are very sensitive functions of the (anti-)neutrino energy, especially for low energies. Thus, extracting any information from the low energy tail of the detector muon spectrum would necessitate very good energy resolution. However, with the neutrino-nucleon cross sections being small for such $E_{\nu}$, these neutrinos have only a relatively small contribution to make to the total number of muon events.

Keeping in mind that $\nu-N$ cross-section is nearly double ${ }^{8}$ that of $\bar{\nu}-N$ we argue that the detector should be exposed to a $\bar{\nu}_{\mu}$ beam for double the time that it is exposed to a $\nu_{\mu}$ beam. To be specific, we consider a $50 \mathrm{kT}$-year exposure to a $\nu_{\mu}$ beam and a $100 \mathrm{kT}$-year exposure to $\bar{\nu}_{\mu}$ beam, or in other words, a total exposure of 3 years for the detector configuration [14] under consideration.

\subsection{The rate asymmetry for $\nu_{\mu}\left(\bar{\nu}_{\mu}\right)$ initiated events}

Armed with the above, we can now calculate the difference in $\mu^{-}$and $\mu^{+}$event numbers. Defining an asymmetry of the form

$$
A_{N} \equiv \frac{N_{-}-N_{+}}{N_{-}+N_{+}}
$$

\footnotetext{
${ }^{8}$ For the total cross section, this ratio has only a mild dependence on the incident neutrino energy, and for the energy range we are interested in has a value close to 2.18. However, on imposition of an energy threshold $E\left(\mu^{ \pm}\right)>2 \mathrm{GeV}$ that such a detector is expected to have, the ratio is a more sensitive function of the (anti-)neutrino energy (see Fig. 6). In our numerical analysis, we explicitly account for this factor and the consequent effect on the error bars.
} 
we present, in Fig. 7, the same for a baseline of $4865 \mathrm{~km}$. The values for $\left(\left|U_{\mu 3}\right|^{2},\left|U_{e 3}\right|^{2}\right)$ correspond to the two extreme cases of Ref. [15]. Also plotted are the analogous expectations for $\left|U_{\mu 3}\right|^{2}=0.5$, a case that, to the lowest order, would have been associated with vanishing $A_{N}$. We have binned the expected data, keeping under consideration both the expected energy
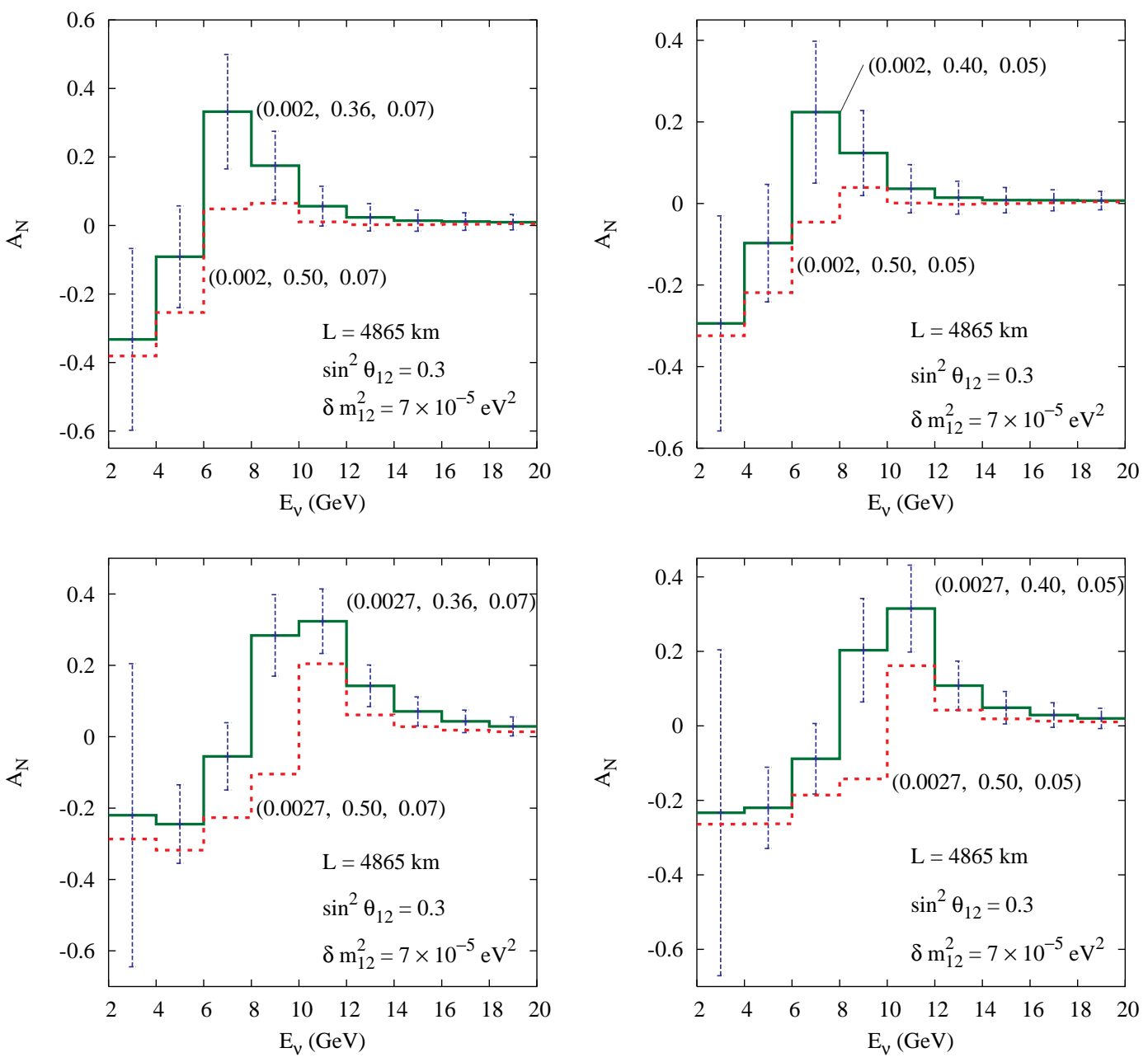

Figure 7: The difference between $\nu_{\mu}$ and $\bar{\nu}_{\mu}$ charged-current events for a baseline $L=4865$ $\mathrm{km}$ with an integrated luminosity of $10^{20}$ muons and double the number of antimuons. The detector is a $50 \mathrm{kT}$ iron calorimeter with a muon energy threshold of $2 \mathrm{GeV}$ [14]. Each plot corresponds to a different combination of $\left(\delta m_{13}^{2},\left|U_{\mu 3}\right|^{2},\left|U_{e 3}\right|^{2}\right)$. The other parameters are: $\delta m_{12}^{2}=7 \times 10^{-5} \mathrm{eV}^{2}$ and $\sin ^{2} \theta_{12}=0.3$. Also shown are the $1 \sigma$ error bars.

resolution [14] as well as the number of events in a particular bin. Also shown are the statistical $1 \sigma$ error bars. Several features are easily discernible:

- In each of the cases displayed, the rate asymmetry is statistically significant in more than one bin. Together, they lead to a very considerable shift in the $\chi^{2}$. This amply demonstrates the sensitivity of such an experiment to a departure from maximality of $U_{\mu 3}$.

- As expected, the signal is less pronounced for the case $\left(\left|U_{\mu 3}\right|^{2},\left|U_{e 3}\right|^{2}\right)=(0.40,0.05)$ than for $(0.36,0.07)$. This is just a reflection of the fact - see eq.(9) - that the rate asymmetry is proportional to $\left|U_{e 3}\right|^{2}$. While the proportionality to $\left(1-2\left|U_{\mu 3}\right|^{2}\right)$ is no longer an accurate statement, it, nevertheless still encapsulates a large measure of truth. 
- In the energy range of interest, the binwise asymmetry clearly shows an oscillatory behaviour, a feature that would have been absent for a similar detector had the baseline been shorter than $\sim 1000 \mathrm{~km}$.

- The functional dependence of $\Delta N\left(E_{\nu}\right)$ exhibits a discernible dependence on the mixing angles $\left(\left|U_{\mu 3}\right|^{2},\left|U_{e 3}\right|^{2}\right)$, and could, in principle, be used to distinguish between values for these parameters. However, to obtain a significant resolution in the parameter space, a much larger event count would be necessary.

- The dependence on the value of $\delta m_{13}^{2}$, on the other hand, is much more pronounced. The shift in $\Delta P$ that we encountered in Fig.3 is quite well reflected by a shift in $A_{N}$, even after the convolution with the muon spectrum and the energy-dependent cross sections as well as the finite resolution effects. Thus, our previous comment about using this measurement for a determination of $\delta m_{13}^{2}$ is substantiated.

- The energy dependence is quite different for the two baselines considered and can be used to advantage. For example, if storage rings come up at both the JHF [20] and Fermilab, a detector such as ICAL/INO [14] could use beams from both to distinguish more efficiently between the possible parameter sets.

\subsection{Wrong sign muons}

We now turn to a discussion $\nu_{e}$ to $\nu_{\mu}$ conversion (as well the conjugate process) and their detection in the iron calorimeter detector under consideration. Such events are obviously characterised by the appearance of a muon with a charge opposite to that of the decaying particle in the storage ring. Since the detector is to be magnetized one, charge measurement is relatively straightforward thus rendering these events easily distinguishable. Efficacy of 'wrong-sign-muon' events in measuring the angle $\theta_{13}$ has already been advocated in Ref. [10] in the context of muon-storage ring neutrino factory experiments. Here we want to emphasise that how an independent measurement of 'wrong-sign-muon' events can also supplement the more accurate measuremeant of $\theta_{23}$. We have already seen, vide Fig.5, that the conversion probability for $\nu_{e}$ is typically larger than that for $\bar{\nu}_{e}$, a consequence of the sign of $\delta m_{12}^{2}$. This fact, alongwith the smaller detection efficiency for $\bar{\nu}_{\mu}$, implies that it is wiser to concentrate on the $\mu^{+} \rightarrow \nu_{e} \rightarrow \nu_{\mu}$ chain rather than attempt to measure the conjugate process as well. And since we have already advocated twice as long an exposure to $\mu^{+}$decays as compared to $\mu^{-}$ decays, this works in our favour too.

In Fig.8, we present the number of $\mu^{-}$events in the detector when exposed to $\mu^{+}$decays. As before, the detector is supposed to be a $50 \mathrm{kT}$ one, with a total exposure of $2 \times 10^{20}$ decays. Clearly, the number of events grows with $\left|U_{e 3}\right|^{2}$. While the exact dependence is not linear (as the naive expectation would be), explicit calculations with other values of $\left|U_{e 3}\right|$ shows that it is not very far from being linear. This is also the case for the larger baseline of $10480 \mathrm{~km}$ (which we do not exhibit here). The dependence on $\left|U_{\mu 3}\right|$ is more complicated though. As can be expected, this depends more crucially on the size of the matter effect and hence on the exact baseline (see, for example, Fig. 5). However, given the considerably large number of such events, it is quite apparent that this measurement is likely to be a sensitive probe in the $\left(\left|U_{\mu 3}\right|,\left|U_{e 3}\right|\right)$ plane. Unfortunately though, the event distribution is not very sensitive to $\delta m_{13}^{2}$.

The main motivation for the above excercise is to show that the measurement of any of the small parameters $\left(\left|U_{e 3}\right|\right.$ or $\left.\beta\right)$ at long baseline experiments cannot be done independent of each 

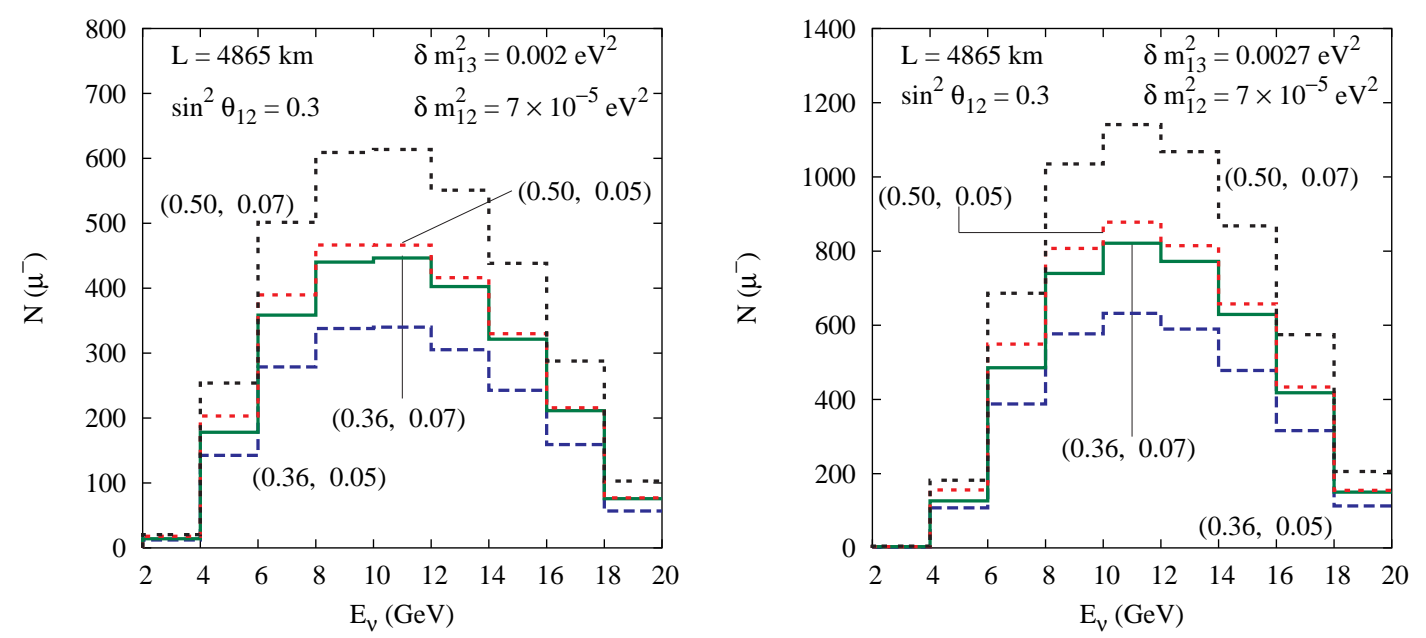

Figure 8: The number of $\mu^{-}$events in a $50 \mathrm{kT}$ iron calorimeter exposed to a total of $2 \times 10^{20} \mu^{+}$ decays in a $20 \mathrm{GeV}$ storage ring at a distance of $4865 \mathrm{~km}$. The histograms correspond to different values of $\left(\left|U_{\mu 3}\right|^{2},\left|U_{e 3}\right|^{2}\right)$ with the left (right) panels referring to $\delta m_{13}^{2}=0.002(0.0027) \mathrm{eV}^{2}$.

other. Although experiments with relatively small baselines (such as NuMi-MINos) seem to be quite sensitive to the parameter $\beta$, this optimism is misplaced as explained in Sections $3 \& 4$. On the other hand, moderate baseline experiments do show increased sensitivity to $\beta$, but only at the cost of having a more complicated dependence on $\beta$ and $\left|U_{e 3}\right|$ (see eq.14 and Fig. 4). In either case, a measurement of $\beta$ from $\nu_{\mu}$-survival probability asymmetry needs an accurate knowledge of $\left|U_{e 3}\right|$. It is here that the wrong-sign muon rates (which is more sensitive to $U_{e 3}$ ) have the most important role to play. Of course, a precise knowledge of $\delta m_{13}^{2}$ is important for the extraction of $\left|U_{e 3}\right|$ (although the dependence on $\delta m_{13}^{2}$ is not as pronounced for the wrong-sign muon events as for the right-sign ones). However, it is expected that the error-bars on $\delta m_{13}^{2}$ would be significantly reduced before the era of such neutrino factory experiments. Then, in principle, $A_{N}$ and wrong-sign muon rates could be used simultaneously in a statistical analysis (in a spirit similar to that followed in Ref. [11]) to extract $\left|U_{e 3}\right|$ and $\beta$ to much greater accuracy than possible today.

\section{Conclusions}

Analyses of matter effects in $\nu_{\mu}$ oscillations have largely concentrated on transition probabilities. Revisting the problem, we demonstrate that the survival probabilities too are very sensitive to matter effects and can be used profitably in determination of several crucial parameters in the neutrino sector.

To start with, we derive a set of approximate analytical expressions for the $\nu_{\mu}$ survival probability in presence an arbitrarily large matter density. While similar results had already been reported for small matter terms in the effective Hamiltonian, our expressions have a much wider range of validity.

Since we are interested in the survival probability for $\nu_{\mu}$, a variable of interest can be constructed simply from the number of charged current (CC) events in the detector. Starting with $\nu_{\mu}$ and $\bar{\nu}_{\mu}$ beams, $A_{N} \equiv\left(N_{-}-N_{+}\right) /\left(N_{-}+N_{+}\right)$describes an asymmetry between the number of 'same-sign' $\mu^{-}$and $\mu^{+}$events generated in the detector after traversing a given baseline. We show by explicit event rate calculation that this asymmetry is a good measure of 
the matter effects felt by the neutrinos while propagating through the earth. However, contrary to claims in the literature [15], the size of the asymmetry for the Fermilab-MINos combine is much smaller than the experimental sensitivity.

We suggest, therefore, that a future long-baseline experiment could explore this effect. As a prototype experiment, we consider a neutrino factory (a muon storage with muon beams of energy $20 \mathrm{GeV}$ ) and the proposed $50 \mathrm{kT}$ iron calorimeter detector $[13,14]$ with a capability of muon charge determination. Using realistic experimental resolutions, we demonstrate that such a combination is sufficient to establish the aforementioned asymmetry with a very large statistical significance. However, unlike in the case of relatively short-baseline Fermilab-MINOS combine, the asymmetry for a long baseline is no longer proportional to the deviation of $\left|U_{\mu 3}\right|^{2}$ from maximality. Even so, the sensitivity to $\left(1-2\left|U_{\mu 3}\right|^{2}\right)$ remains quite pronounced for baselines of upto about $6500 \mathrm{Km}$. Using the aforementioned approximate expressions for the survival probabilities, such data can thus be used to determine $\left(1-2\left|U_{\mu 3}\right|^{2}\right)$. While the sensitivity to this combination decreases for much longer baselines, asymmetry data for such baselines can still be used for precise determination of $\left|U_{e 3}\right|$.

The latter exercise is also shown to be aided by the measurement of 'wrong-sign-muon' events generated through $\nu_{e} \rightarrow \nu_{\mu}$ oscillations. Estimating the rate of such events for the same experimental setup, we demonstrate that this could be used in conjunction with the asymmetry measurement to lead to a good simultaneous determination of $\left|U_{\mu 3}\right|$ and $\left|U_{e 3}\right|$.

\section{Acknowledgements}

The authors thank the Theory Division, CERN for hospitality during the initiation phase of the project. DC thanks INFN, Sezione di Roma, for hospitality during which a major part of this work has been done. DC acknowledges financial assistance under the Swarnajayanti Fellowship grant from the Department of Science and Technology, India. AD is partially supported by the RTN European Programme MRTN-CT-2004-503369 (Quest for Unification).

\section{References}

[1] Super-Kamiokande collaboration, Y. Hayato, talk given at the EPS 2003 conference (Aachen, Germany, 2003), http://eps2003.physik.rwth-aachen.de;

S. Fukuda et al., Phys. Lett. B539, 179 (2002).

[2] SNO collaboration, S.N. Ahmed et al., nucl-ex/0309004.

[3] CHOOZ collaboration, M. Appolonio et al., Eur. Phys. J C27, 331 (2003).

[4] KamLAND collaboration, K. Eguchi et al., Phys. Rev. Lett. 90, 021802 (2003).

[5] K2K collaboration, I. Kato, talk given at the 38th Recontres de Moriond on Electroweak Interactions and Unified Theories (Les Ares, France, 2003), hep-ex/0306043.

[6] For a recent review for the limits on masses and mixing, see S. Goswami, A. Bandyopadhyay and S. Choubey, hep-ph/0409224.

[7] L. Wolfenstein, Phys. Rev. D17, 2369 (1978);

S. Mikheyev and A. Smirnov, Sov. J. Nucl. Phys. 42, 913 (1985). 
[8] For example, see S. Geer, FERMILAB-CONF-04-133-E, 2004;

R. Raja,. FERMILAB-CONF-04-018-E (2004), hep-ex/0402022;

A. Blondel et. al., Nucl.Instrum. Meth. A451,102 (2000).

[9] V. Barger, S. Geer, R. Raja and K. Whisnanat, Phys. Rev. D62, 013004 (2000);

C. Albright et al., hep-ex/0008064;

M. Freund, P. Huber and M. Lindner, Nucl. Phys. B585 105 (2000); Nucl. Phys. B615 331 (2001);

M. Apollonio et al., hep-ph/0210192.

[10] V. Barger, S. Geer, R. Raja and K. Whisnanat, Phys.Lett. B485 379 (2000);

A. Cervera et al., Nucl. Phys. B579 17 (2000).

[11] M. Aoki et al., Phys. Rev. D67, 093004 (2003);

M. Aoki, K, Hagiwara and N. Okamura, hep-ph/0311324.

[12] R. Gandhi et al., hep-ph/0408361.

[13] N.Y. Agafonova et al. [MONOLITH Collaboration], LNGS-P26-2000, LNGS-P26-00, CERN-SPSC-2000-031, CERN-SPSC-M-657; see http://castore.mi.infn.it/ monolith/

[14] See http://www.imsc.res.in/ ino; and working reports and talks therein.

[15] S. Choubey and P. Roy, Phys. Rev. Lett. 93, 021803 (2004).

[16] A. M. Dziewonski and D.L. Anderson, Phys. Earth Plan. Int. 25, 297 (1981);

we use the parametrisation given in R. Gandhi, C. Quigg, M. Reno and I. Sarcevic, Astropart. Phys. 5, 81 (1996).

[17] E. Akhmedov et al., Jour. High Energy Phys. 0404:078 (2004) .

[18] R. Saakian et al (MINOs collaboration), Nucl. Phys. Proc. Suppl. 111, 169 (2002); Yad.Fiz. 67, 1112 (2004);

M.V. Diwan et al., Nucl. Phys. Proc. Suppl. 123, 272 (2003).

[19] MINERvA Collaboration (D.A. Harris for the collab.), FERMILAB-PUB-04-252-E, hepex/0410005;

[20] Y. Itow et al., hep-ex/0106019; also see : http://neutrino.kek.jp/jhfnu. 